\title{
Sodium valproate, a histone deacetylase inhibitor, modulates the vascular endothelial growth inhibitor-mediated cell death in human osteosarcoma and vascular endothelial cells
}

\author{
KOJI YAMANEGI $^{1 *}$, MUTSUKI KAWABE $^{1,2^{*}}$, HIROYUKI FUTANI $^{3}$, HIROSHI NISHIURA $^{1}$, NAOKO YAMADA $^{1}$, \\ NAHOKO KATO-KOGOE $^{1}$, HIROMITSU KISHIMOTO ${ }^{2}$, SHINICHI YOSHIYA $^{3}$ and KEIJI NAKASHO ${ }^{1}$ \\ Departments of ${ }^{1}$ Pathology, ${ }^{2}$ Oral and Maxillofacial Surgery and ${ }^{3}$ Orthopedic Surgery, \\ Hyogo College of Medicine, Nishinomiya, Hyogo 663-8501, Japan
}

Received December 29, 2014; Accepted February 12, 2015

DOI: $10.3892 /$ ijo.2015.2924

\begin{abstract}
The level of vascular endothelial growth inhibitor (VEGI) has been reported to be negatively associated with neovascularization in malignant tumors. The soluble form of VEGI is a potent anti-angiogenic factor due to its effects in inhibiting endothelial cell proliferation. This inhibition is mediated by death receptor 3 (DR3), which contains a death domain in its cytoplasmic tail capable of inducing apoptosis that can be subsequently blocked by decoy receptor 3 (DcR3). We investigated the effects of sodium valproate (VPA) and trichostatin A (TSA), histone deacetylase inhibitors, on the expression of VEGI and its related receptors in human osteosarcoma (OS) cell lines and human microvascular endothelial (HMVE) cells. Consequently, treatment with VPA and TSA increased the VEGI and DR3 expression levels without inducing DcR3 production in the OS cell lines. In contrast, the effect on the HMVE cells was limited, with no evidence of growth inhibition or an increase in the DR3 and DcR3 expression. However, VPA-induced soluble VEGI in the OS cell culture medium markedly inhibited the vascular tube formation of HMVE cells, while VEGI overexpression resulted in enhanced OS cell death. Taken together, the HDAC inhibitor has anti-angiogenesis and antitumor activities that mediate soluble VEGI/DR3-induced apoptosis via both autocrine and paracrine pathways. This study indicates that the HDAC inhibitor may be exploited as a therapeutic strategy modulating the soluble VEGI/DR3 pathway in osteosarcoma patients.
\end{abstract}

Correspondence to: Dr Koji Yamanegi, Department of Pathology, Hyogo College of Medicine, 1-1 Mukogawa-cho, Nishinomiya, Hyogo 663-8501, Japan

E-mail: yamanegi@hyo-med.ac.jp

*Contributed equally

Key words: valproic acid, HDAC inhibitor, osteosarcoma, vascular endothelial growth inhibitor

\section{Introduction}

Osteosarcoma (OS) is the most common primary malignant tumor of the bone, occurring mainly in children and young adolescents. With combination therapy, the 5-year survival of affected patients is $60-70 \%$. However, the survival rates of patients with metastasis or recurrence are far worse, at $<30 \%$ and $<20 \%$, respectively (1). The lungs are the predominant site of OS metastasis, and pulmonary involvement is the most common cause of death. Unfortunately, the survival rates have not improved over the last 20 years, despite the significant increase in clinical trials.

Epigenetic modifications have been shown to have critical roles in regulating gene expression. DNA methylation and histone modifications are also important mediators of epigenetic gene silencing and essential steps in diverse biological processes (2). Increasing evidence implicates histone deacetylase (HDAC) inhibitors as some of the most promising epigenetic anticancer agents $(3,4)$. Valproic acid (VPA), an HDAC inhibitor, is a well-established long-term treatment for epilepsy and bipolar disorder $(5,6)$. However, in recent years, VPA and trichostatin A (TSA) have also been shown to have the potential ability to modulate the biology of several tumor cell types by inducing differentiation, apoptosis and immunogenicity and subsequently decreasing the rates of metastasis and angiogenesis (3). Angiogenesis is a key biological process that occurs during embryonic development and tissue repair and is required for tumor progression and metastasis. Vascular endothelial growth factor (VEGF), a well-known pro-angiogenic factor, plays an important role in tumor-induced angiogenesis in various human tumors. The tumor angiogenesis mediated by hypoxia inducible factor- $1 \alpha(\mathrm{HIF}-1 \alpha)$ and its transcriptional target, VEGF, is increased, and the anti-angiogenic function of HDAC inhibitors is to suppress the VEGF gene expression via HIF-1 $\alpha$ inhibition (7).

Vascular endothelial growth inhibitor (VEGI), also known as TL1A or TNFSF15, is a novel member of the tumor necrosis factor (TNF) superfamily (8). VEGI was originally thought to be exclusively expressed in endothelial cells and abundantly expressed in the kidneys, lungs, prostate, placenta and liver $(8,9)$. However, it was subsequently reported that VEGI is 
also expressed in a wide variety of human cancer cell lines, including breast, prostate, bladder, colorectal and liver cancers (10). VEGI contains 251 amino acids in the full-length product of TNF ligand-related molecule 1 (TL1), and three isoforms of VEGI have been reported, all sharing a common 151 C-terminal amino acid sequence with different N-terminal regions $(11,12)$. The biological activity of VEGI is dependent on the solubilized extracellular domain of these isoforms, and the secreted or recombinant soluble forms of VEGI are potent inhibitors of endothelial cell proliferation and angiogenesis as well as tumor growth and neovascularization $(9,11,13-15)$. This inhibition is mediated by death receptor 3 (DR3) (TNFRSF25), known to be a functional receptor for VEGI $(11,16)$, which contains a death domain in its cytoplasmic tail capable of inducing apoptosis that can be blocked by decoy receptor 3 (DcR3) (12). DcR3 is a soluble receptor lacking transmembrane domains that belongs to the TNFR superfamily. The overexpression of DcR3 noted in the some malignant cells blocks the suppression of apoptosis by interfering with the binding of VEGI, FasL and LIGHT to receptors $(12,17)$. In addition, DcR3 enhances angiogenesis by blocking the antiangiostatic function of VEGI (17).

In this study, we investigated the effects of HDAC inhibitors on the endogenous anti-angiogenic factor VEGI and its receptors and found that treatment with VPA and TSA increased the expression of VEGI and its receptor, DR3, without inducing increased DcR3 in OS cells. Moreover, VPA-treated OS cell culture medium containing soluble VEGI inhibited vascular tube formation, while the overexpression of VEGI in the OS cells induced cell death. Therefore, HDAC inhibitors result in enhanced tumor and vascular endothelial cell death via the soluble VEGI/DR3 autocrine- and paracrine pathways.

\section{Materials and methods}

Reagents and antibodies. Sodium valproate (VPA) and trichostatin A (TSA) were purchased from Wako (Osaka, Japan), anti-acetyl-histone $\mathrm{H} 3$ rabbit polyclonal antibodies were purchased from Upstate (Temecula, CA, USA), anti-human VEGI mouse monoclonal antibody $\left(\mathrm{IgG}_{1}\right)$, anti-human DR3 mouse monoclonal antibody $\left(\operatorname{IgG}_{2 b}\right)$ and horseradish peroxidase-conjugated goat anti-rabbit $\mathrm{IgG}_{1}$ antibody were purchased from Santa Cruz (Santa Cruz, CA, USA), biotin-conjugated anti-human VEGI rabbit polyclonal antibodies (IgG) and anti-human VEGI rabbit polyclonal antibodies ( $\mathrm{IgG}$ ) were purchased from Abcam (Tokyo, Japan) and anti-actin rabbit polyclonal antibodies and horseradish peroxidase-conjugated rabbit anti-mouse IgG antibodies were purchased from Sigma (St. Louis, MO, USA).

Cells. U-2 OS and SaOS-2 human osteosarcoma cells were purchased from the American Type Culture Collection (ATCC, Manassas, VA, USA), and Riken BRC Cell Bank (Tsukuba, Ibaragi, Japan), respectively. The U-2 OS and SaOS-2 cells were cultured in McCoy's 5A modified medium (Invitrogen, Carlsbad, CA, USA). Primary normal human dermal microvascular endothelial (HMVE) cells were purchased from the Cell Systems Corp. (CSC, Kirkland, WA, USA) and cultured using the CS-C medium kit (DS Pharma Biomedical, Osaka, Japan). All media contained 10\% fetal bovine serum (FBS)
(MP Biomedical; Morgan Irvine, CA, USA), penicillin $(100 \mathrm{U} / \mathrm{ml})$ and streptomycin $(100 \mu \mathrm{g} / \mathrm{ml})$, and all cells were cultured in a humidified atmosphere of $5 \% \mathrm{CO}_{2}$ in air at $37^{\circ} \mathrm{C}$.

Reverse transcription polymerase chain reaction (RT-PCR). Total RNA was extracted from the U-2 OS, SaOS-2 and HMVE cells using TRIzol reagent (Invitrogen) according to the manufacturer's instructions. Reverse transcription of $2 \mu \mathrm{g}$ of total RNA was performed at $42^{\circ} \mathrm{C}$ for $1 \mathrm{~h}$ using random primer (Roche Diagnostics, Tokyo, Japan) and reverse transcriptase (Roche Diagnostics), and the cDNAs thus produced were sequentially amplified by PCR with Takara Ex Taq ${ }^{\mathrm{TM}}$ DNA polymerase (Takara Bio, Ohtsu, Shiga, Japan) using the following specific primer sets: sense, 5'-CTCTGCACTG GGAACATGAA-3' and antisense, 5'-TTGGCTCAGGGTA GCTGTCT-3' for VEGI; sense, 5'-CGCAGA GATACTGACTG TGG-3' and antisense, 5'-AGGAGGTGCTAGAAGGGTGT-3' for DR3; sense, 5'-GCTACTGCAACGTCCTCTG-3' and antisense, 5'-ACACTCCTCAGCTCCTGGTA-3' for DcR3; sense, 5'-GTCATCAATGGAAATCCCATCACC-3' and antisense, 5'-GCTCAGGGATGACCTTGCCC-3' for GAPDH. The amplification conditions for PCR of VEGI, DR3 and DcR3 included 35 cycles at $95^{\circ} \mathrm{C}$ for $30 \mathrm{sec}, 57^{\circ} \mathrm{C}$ for $1 \mathrm{~min}$ and $72^{\circ} \mathrm{C}$ for $1 \mathrm{~min}$, followed by heating at $72^{\circ} \mathrm{C}$ for $7 \mathrm{~min}$, while that for GAPDH included 30 cycles at $95^{\circ} \mathrm{C}$ for $30 \mathrm{sec}, 57^{\circ} \mathrm{C}$ for $30 \mathrm{sec}$ and $72^{\circ} \mathrm{C}$ for $1 \mathrm{~min}$, followed by heating at $72^{\circ} \mathrm{C}$ for $7 \mathrm{~min}$. The amplified fragments were resolved by electrophoresis in $1.5 \%$ agarose gel and detected using ethidium bromide staining.

Quantitative real-time polymerase chain reaction (real-time PCR). U-2 OS, SaOS-2 cells and HMVE cells were cultured in medium with or without $1.0 \mathrm{mM}$ VPA and $30 \mathrm{nM}$ TSA with a change of the medium on day 3. Total RNA was extracted from the cells in each culture dish on days 3 and 7 using TRIzol reagent (Invitrogen). An aliquot of RNA was reverse transcribed using the High Capacity RNA to cDNA kit (Applied Biosystems, Foster City, CA, USA), according to the manufacturer's instructions, and real-time PCR for VEGI, DR3 and DcR3 mRNAs was performed using TaqMan Gene Expression assays (Applied Biosystems). The primer set was Hs00270802_ ml for VEGI mRNA, Hs00600930_ml for DR3 mRNA and Hs00187070_ml for DcR3 mRNA, respectively (Applied Biosystems). The amount of GAPDH mRNA was estimated as an internal reference using human GAPDH endogenous control (Applied Biosystems), and the amount of VEGI, DR3 and DcR3 mRNA in each sample was corrected for the amount of GAPDH mRNA in corresponding samples. The expression levels of each gene in the treated cultures are expressed as the ratio of the average value in the control cultures.

Enzyme-linked immunosorbent assay (ELISA). U-2 OS, SaOS-2 and HMVE cells were seeded at $2 \times 10^{5}$ cells/dish in $10-\mathrm{cm}$ tissue culture dishes containing $5 \mathrm{ml}$ of medium per dish. After $24 \mathrm{~h}$ (day 0$)$, VPA $(1.0 \mathrm{mM})$ or TSA $(30 \mathrm{nM})$ was added to the medium, and the cells were cultured for 7 days with a change of the medium on day 3. After the culture, medium was collected for assays of the soluble VEGI and DcR3. For the detection of soluble VEGI, briefly, 96-well plates were coated with capture antibody, anti-human VEGI mouse monoclonal antibody $(2.0 \mu \mathrm{g} /$ $\mathrm{ml}$ ), overnight at room temperature and then washed three times 
with washing buffer (PBS containing $0.05 \%$ Tween-20) after each incubation period. A standard protein, the recombinant human TL1A/TNFSF15 (R\&D Systems, Minneapolis, MN, USA), and the samples were incubated in the wells for $2 \mathrm{~h}$ at room temperature. After washing, biotin-conjugated anti-human VEGI rabbit polyclonal antibodies $(0.5 \mu \mathrm{g} / \mathrm{ml})$ were incubated in the wells for $2 \mathrm{~h}$ at room temperature. Following horseradish peroxidase-conjugated streptavidin (R\&D Systems) incubation, the substrate solution (R\&D Systems) was subsequently incubated in the wells for $20 \mathrm{~min}$ at room temperature. The detection of soluble DcR3 was performed using the ELISA development system (R\&D Systems), according to the manufacturer's instructions. In order to determine the optical density in each well, the absorbance at $450 \mathrm{~nm}$ against a reference wavelength of $570 \mathrm{~nm}$ was measured using a microplate reader (Bio-Rad, Tokyo, Japan). The effects of VPA and TSA were evaluated by determining the amount of soluble forms per $10^{4}$ viable cells in the treated cultures and expressed as the ratio of the average value in the untreated control cultures.

Western blot analysis. U-2 OS cells cultured with or without VPA or TSA and pVEGI-transfected cells were washed twice with PBS and lysed in radioimmunoprecipitation assay (RIPA) buffer [50 mM Tris- $\mathrm{HCl}$ (pH 7.4), $150 \mathrm{mM} \mathrm{NaCl,} 1 \%$ NP-40, $0.5 \%$ sodium deoxycholate, $0.1 \% \mathrm{SDS}$ ] supplemented with a complete protease inhibitor cocktail (Roche Diagnostics). Briefly, the cell lysates were first incubated on ice for $30 \mathrm{~min}$ and then sonicated several times prior to centrifugation for $10 \mathrm{~min}$ at 10,000 rpm. The supernatant was obtained and analyzed for the protein concentrations according to the Bradford method, with bovine serum albumin as the standard. An aliquot of the supernatant (equivalent to $30 \mu \mathrm{g}$ of proteins) was mixed with a 3 -fold volume of the SDS sample buffer (BioLab, Beverly, MA, USA) containing $10 \% \beta$-mercaptoethanol and subsequently heated at $95^{\circ} \mathrm{C}$ for $10 \mathrm{~min}$, then electrophoresed on $4-12 \%$ Bis-Tris gel with the MES SDS running buffer (Invitrogen) and transferred onto nitrocellulose membranes. The membranes were blocked for $30 \mathrm{~min}$ at room temperature in blocking buffer containing 5\% skim milk in Tris-buffered saline with Tween-20 (TBST) [10 mM Tris, $150 \mathrm{mM} \mathrm{NaCl}$ (pH 7.4), $1 \%$ Tween-20] and then incubated for $90 \mathrm{~min}$ with a 1:500 dilution of anti-acetyl-histone $\mathrm{H} 3$ rabbit polyclonal antibodies, a 1:100 dilution of anti-human VEGI rabbit polyclonal antibodies or a 1:200 dilution of anti-human DR3 mouse monoclonal antibody in TBST buffer, after which the membranes were washed with TBST three times (10 min each). The membranes were then incubated for $90 \mathrm{~min}$ at room temperature with a 1:10,000 dilution of horseradish peroxidase-conjugated goat anti-rabbit IgG antibodies for acetyl-histone H3 or VEGI and horseradish peroxidase-conjugated rabbit anti-mouse $\operatorname{IgG}_{2 b}$ antibody for DR3 and subsequently detected and visualized using the SuperSignal West Pico chemiluminescence detection system (Pierce, Rockford, IL, USA). The same membranes were stripped in stripping buffer (Pierce) and reprobed using the action on the membrane detected with anti-actin rabbit polyclonal antibodies at 1:200 dilution in TBST buffer.

Plasmid construction and transfection. In order to construct a VEGI cDNA plasmid (pVEGI), the RT-PCR fragment amplified from the U-2 OS total RNA using the primer sets sense,
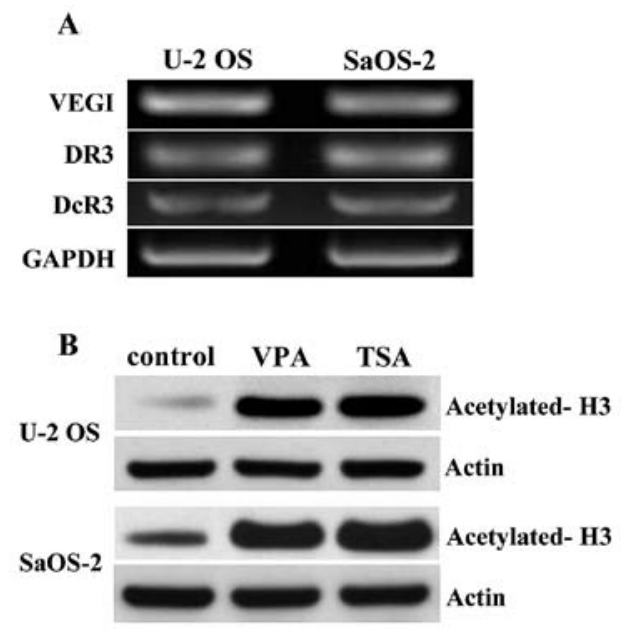

Figure 1. Expression of VEGI and its related receptors in the OS cell lines. (A) Detection of the mRNA transcript using RT-PCR in the OS cell lines. (B) OS cells were cultured for 7 days with or without $1.0 \mathrm{mM}$ VPA or $30 \mathrm{nM}$ TSA and analyzed for the acetylated histone $\mathrm{H} 3$ expression using western blot analysis. The control indicates the absence of both VPA and TSA.

5'-caccATGGCCGATCTGGGA-3' and antisense, 5'-CTATAG TAAGAAGGCTCCAAAGAAGGT-3' was cloned. Following gel purification (Promega, Madison, WI, USA), the amplified DNA fragment was inserted into an expression vector, pcDNA3.1/V5-His-TOPO (Invitrogen). The plasmid inserts were subsequently verified by sequencing. The siRNA designed for the VEGI mRNAs was 5'-ACCUGACAGUUG UGAGACAtt-3' (sense strand), synthesized by Applied Biosystems. For transfection, U-2 OS cells were seeded at $1 \times 10^{5}$ cells/well in 6-well tissue culture plates and cultured in $2 \mathrm{ml}$ of medium for $24 \mathrm{~h}$. The culture medium was changed to Opti-MEM medium (Invitrogen), and the cells were transfected with $1 \mu \mathrm{g}$ of plasmid DNA or $20 \mathrm{nM}$ of siRNA using Lipofectamine ${ }^{\mathrm{TM}} 2000$ (Invitrogen) and RNAiMAX (Invitrogen), respectively, according to the manufacturer's instructions. Total RNA, protein and cell culture media were prepared $48 \mathrm{~h}$ after transfection. The RNA was examined by RT-PCR and real-time PCR, and the proteins were evaluated using western blot analysis. The cell culture medium was assessed according to an ELISA assay in order to determine the soluble VEGI and DcR3 levels, as described above. Images of the transfected OS cells were examined using an inverted microscope (Nikon, Tokyo, Japan).

Human microvascular endothelial (HMVE) cell proliferation assay. HMVE cells $\left(1 \times 10^{3}\right.$ cells per well) were seeded in 96-well tissue culture plates containing $100 \mu \mathrm{l}$ of CS-C complete medium in each well. After $24 \mathrm{~h}$ (day 0), VPA $(1.0 \mathrm{mM})$ or TSA $(30 \mathrm{nM})$ was added to the medium, and the cells were cultured for another 7 days with a change of the medium on day 3 . The number of viable cells in each well was estimated using a CellTiter $96^{\circledR}$ AQueous One solution cell proliferation assay kit (Promega), according to the manufacturer's instructions and presented as the ratio to the average level of optical density in the control cultures.

In vitro tube formation assay. HMVE cells were subjected to an in vitro endothelial tube formation assay (Cell Biolabs 
A.

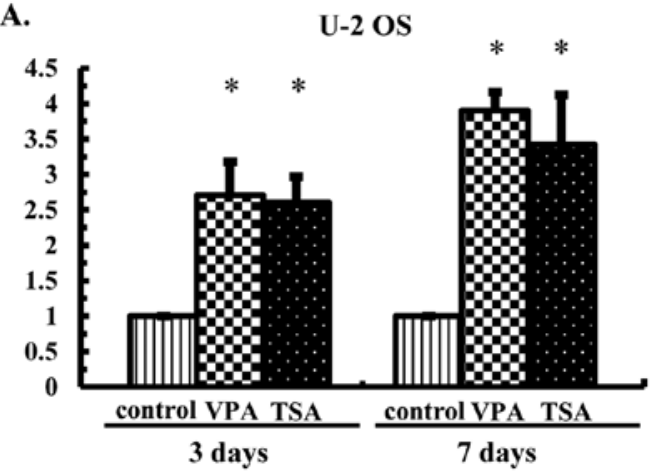

SaOS-2

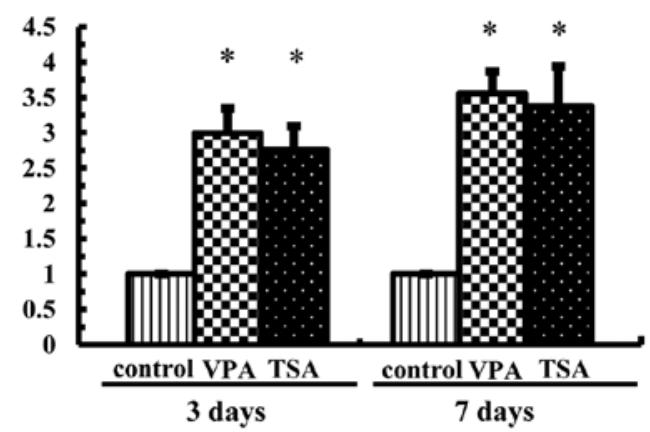

B.

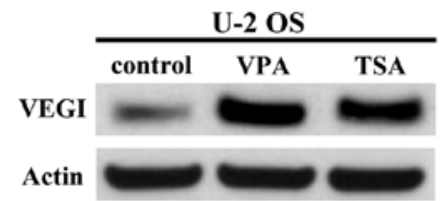

E.

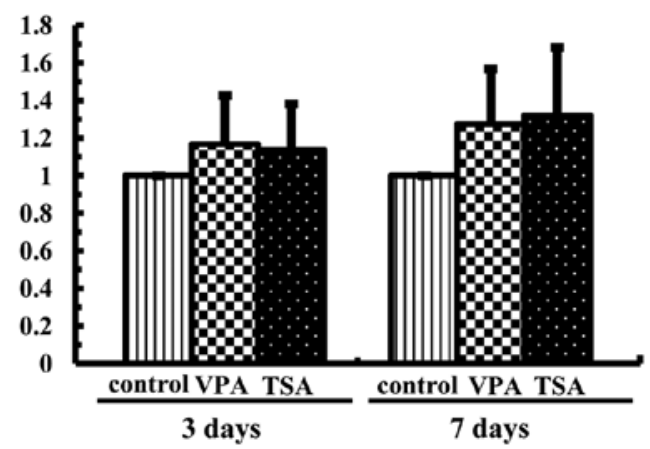

C.
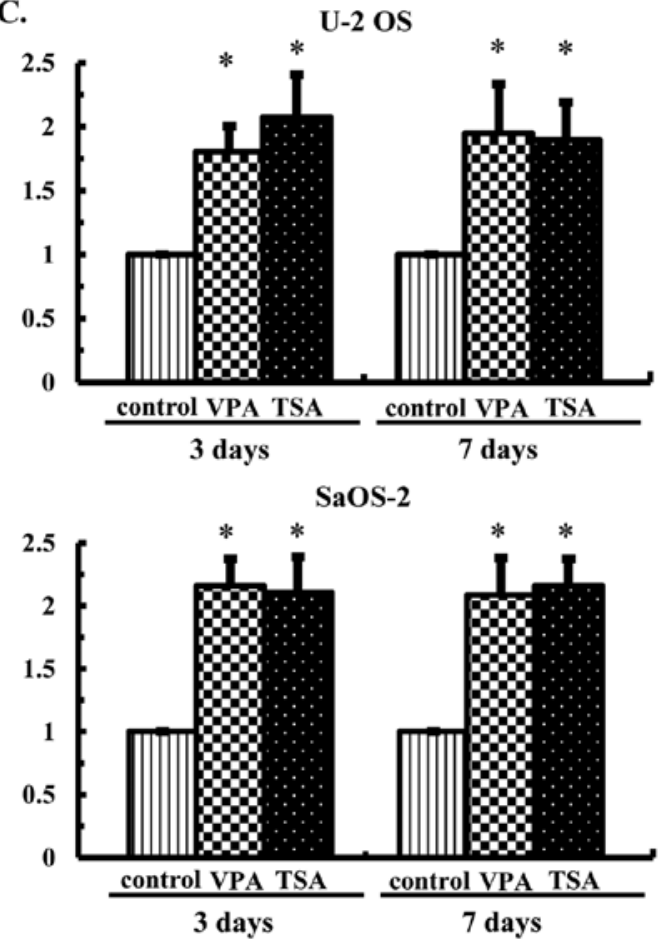

D.
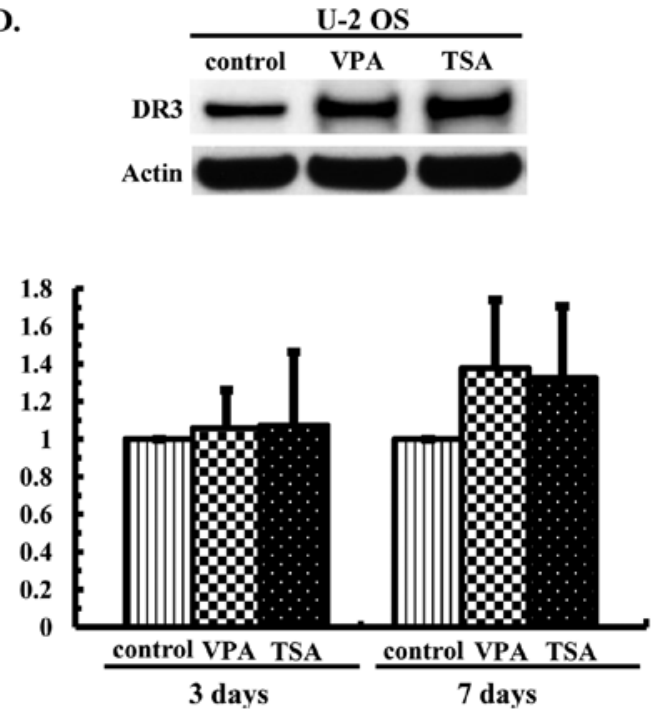

Figure 2. Effects of VPA and TSA on the expression of VEGI and its related receptors in the OS cell lines. U-2 OS and SaOS-2 cells were cultured in medium with or without $1.0 \mathrm{mM}$ VPA or $30 \mathrm{nM}$ TSA for 3 or 7 days. The expression levels of VEGI, DR3 and DcR3 were examined using real-time PCR (A, C and E) The values are expressed as the ratio to the average value in the control cultures. The expression levels of VEGI and DR3 were examined using western blot analysis (B and D). Each bar indicates the mean $\pm \mathrm{SE}$ of values obtained from at least four experiments. ${ }^{*} \mathrm{P}<0.05$, significant difference.

Inc., San Diego, CA, USA), according to the manufacturer's instructions. Briefly, HMVE cells $\left(3.0 \times 10^{3}\right.$ cells) were seeded in ECM gel pre-coated 96-well plates and cultured with HMVE cells in the culture media following the description provided above. After $16 \mathrm{~h}$, the medium was changed to with or without 1.0 mM VPA and VPA-treated or untreated OS cell culture medium, and the culture was extended for additional 16 h. After washing with $1 \mathrm{X}$ staining buffer, Calcein AM staining was performed, the endothelial cells and tubes were examined using a fluorescence microscope (Nikon).

Statistical analysis. The data are presented as the mean \pm SE. Data for three groups or more were analyzed using the two-tailed Dunnett's t-test for multiple comparisons. A P-value of $<0.05$ was considered to be significant.

\section{Results}

Detection of the transcription of VEGI and its related receptors and effects of the HDAC inhibitors on histone acetylation in the OS cells. The expression levels of VEGI, DR3 and DcR3 in OS cells (U-2 OS and SaOS-2 cells) were examined using RT-PCR. All three gene transcripts were detected in the OS cell lines (Fig. 1A). In order to confirm the actions of VPA and TSA as histone deacetylase (HDAC) inhibitors in the OS cells, U-2 OS and SaOS-2 cells were cultured in the presence 

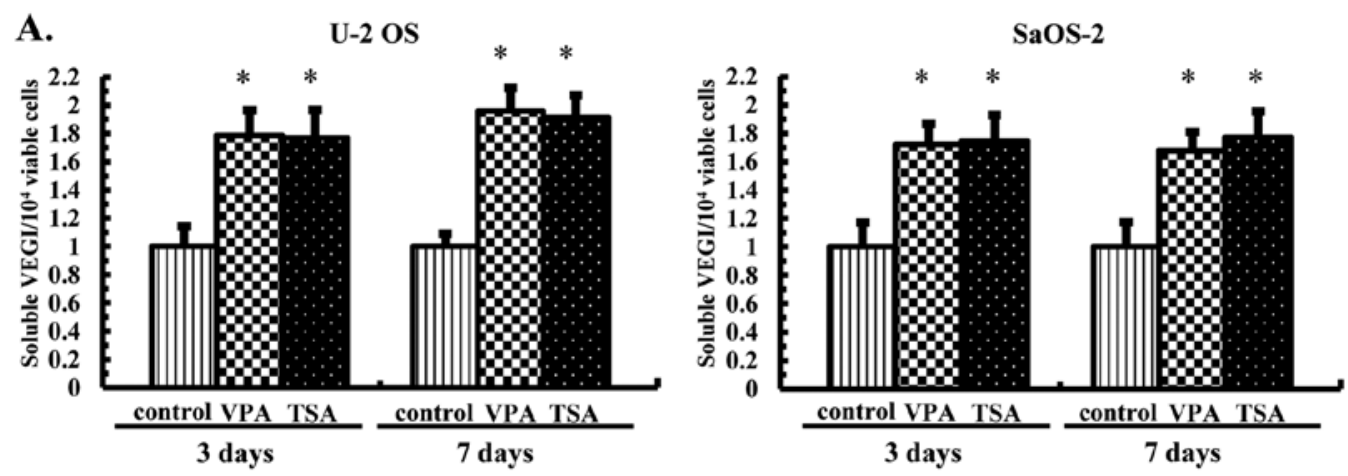

B.

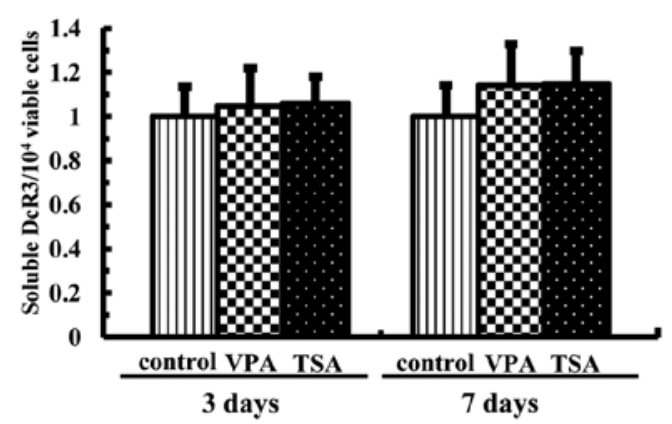

SaOS-2

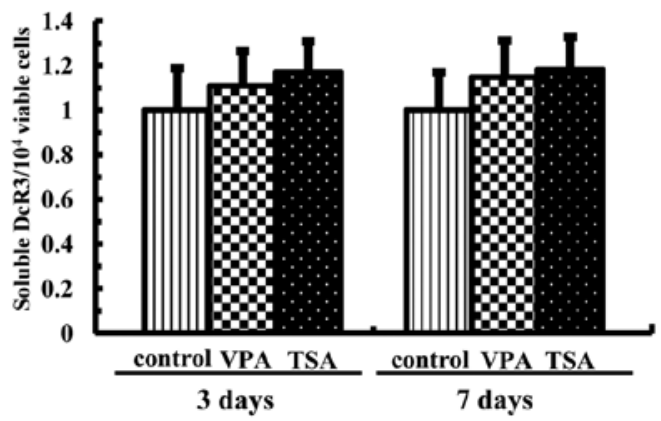

Figure 3. Effects of VPA and TSA on the levels of soluble VEGI and DcR3. U-2 OS and SaOS-2 cells were cultured in medium with or without $1.0 \mathrm{mM}$ VPA and $30 \mathrm{nM}$ TSA for 3 or 7 days. The amount of soluble VEGI (A) and DcR3 (B) in the medium per $10^{4}$ viable cells was determined on days 3 and 7 . The values are expressed as the ratio to the average amount of soluble VEGI and DcR3 per $10^{4}$ viable cells in the control cultures, set at 1 . Each bar indicates the mean \pm SE of values obtained from eight experiments. ${ }^{*} \mathrm{P}<0.05$, significant difference.

or absence of VPA at $1.0 \mathrm{mM}$ or TSA at $30 \mathrm{nM}$ for 7 days, after which the histone $\mathrm{H} 3$ expression was examined using western blot analysis. The results showed significantly increased protein levels of acetylated histone H3 in both the VPA and TSA-treated OS cell lines (Fig. 1B).

Effects of the HDAC inhibitors on the mRNA and protein expression levels of VEGI and its related receptors in the $O S$ cells. U-2 OS and SaOS-2 cells were cultured in the presence or absence of VPA at $1.0 \mathrm{mM}$ or TSA at $30 \mathrm{nM}$ for 3 or 7 days, after which the mRNA expression levels of VEGI, DR3 and DcR3 were examined using real-time PCR and the protein expression levels of VEGI and DR3 were examined using western blot analysis. The mRNA transcription of VEGI was increased 2.6- to 3.7-fold (Fig. 2A), while that of DR3 was increased 1.7- to 2.4-fold (Fig. 2C) in the U-2 OS and SaOS-2 cells, respectively. Increased protein expression levels were also observed (Fig. 2B and D), whereas treatment with VPA or TSA did not significantly increase DcR3 mRNA transcription (Fig. 2E).

Effects of the HDAC inhibitors on the production of soluble VEGI and secretion of DcR3 by the OS cells. In order to determine effects of the HDAC inhibitors on the production of soluble VEGI and secretion of DcR3, U-2 OS and SaOS-2 cells were cultured in the presence of VPA at $1.0 \mathrm{mM}$ or TSA at $30 \mathrm{nM}$ for 3 or 7 days, and the levels of soluble VEGI and DcR3 accumulated in the medium during the first 3 days and the next 4 days were analyzed using ELISA assays. Consequently, the soluble VEGI levels were significantly increased in the OS cells on 3 and 7 days (Fig. 3A). However, the levels of DcR3 remained essentially unchanged in the U-2 OS and SaOS-2 cells at 3 and 7 days following treatment with both HDAC inhibitors (Fig. 3B).

VEGI overexpression directly induces osteosarcoma cell death. Because treatment with VEGI induced apoptosis in the vascular endothelial cells (13), we attempted to confirm whether VEGI caused apoptosis in tumor cells. Either VEGI full-length gene expression or control vectors or siRNA for VEGI were transfected into U-2 OS cells, and the overexpression of VEGI was confirmed based on the protein expression (Fig. 4A). An 4-fold increase in soluble VEGI secretions, without DcR3 productions, was also observed (Fig. 4B). Moreover, the transfection of VEGI siRNA did not change either the soluble VEGI or DcR3 levels (Fig. 4B), whereas the overexpression of VEGI significantly induced OS cell death compared with that seen in the control and siRNA-transfected cells (Fig. 4C).

Transcription of VEGI and its related receptors and effects of the HDAC inhibitors on cell proliferation in the HMVE cells. The expression levels of VEGI, DR3 and DcR3 were examined in the HMVE cells using RT-PCR. All three genes were transcribed in the HMVE cells (Fig. 5A), and an analysis of HMVE cell growth showed that neither treatment with VPA at $1.0 \mathrm{mM}$ or TSA at $30 \mathrm{nM}$ had an effect different from that observed in the control cells (Fig. 5B). However, a real-time 
A.
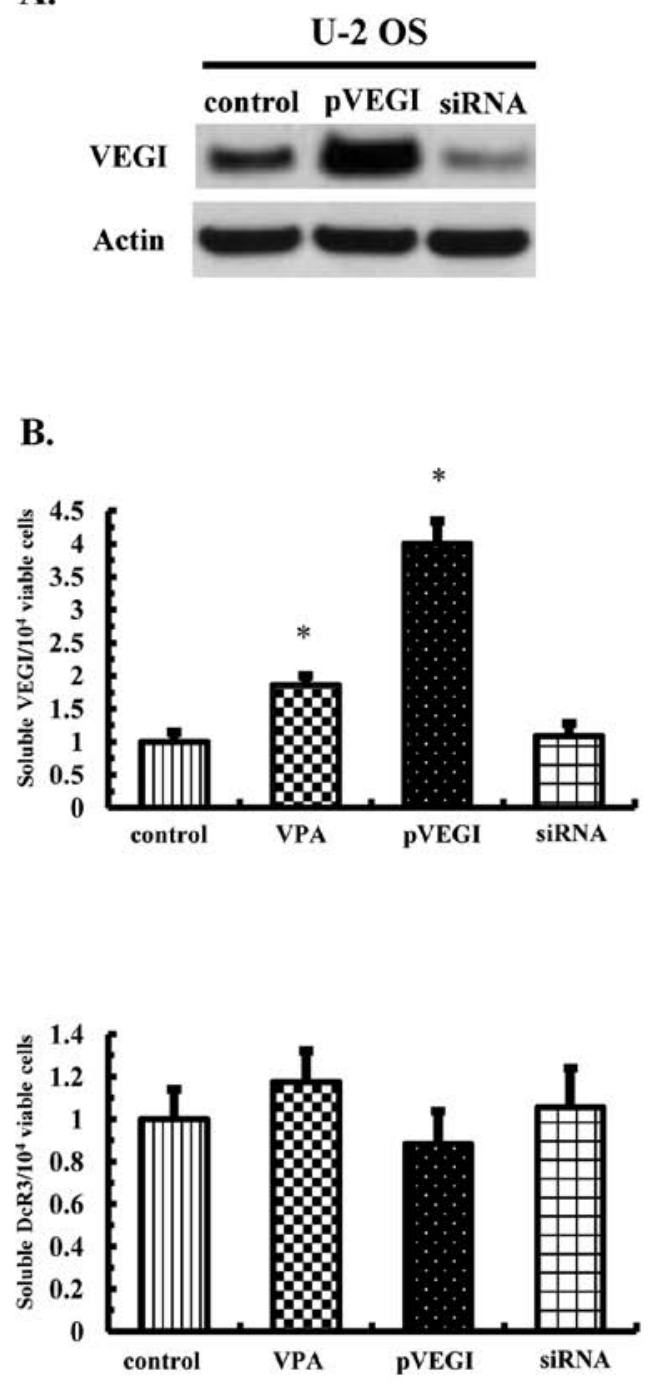

C.

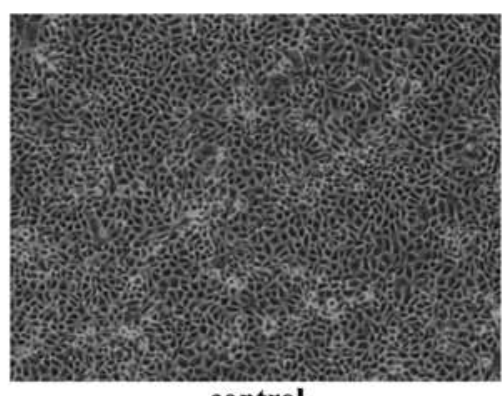

control

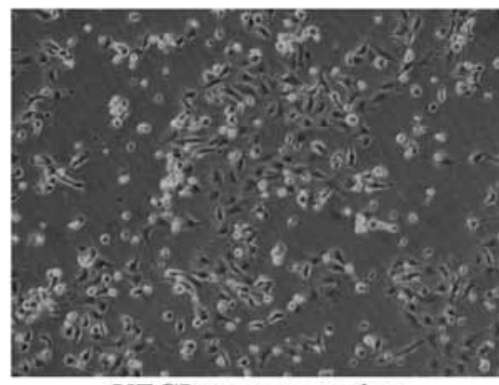

VEGI-overexpression

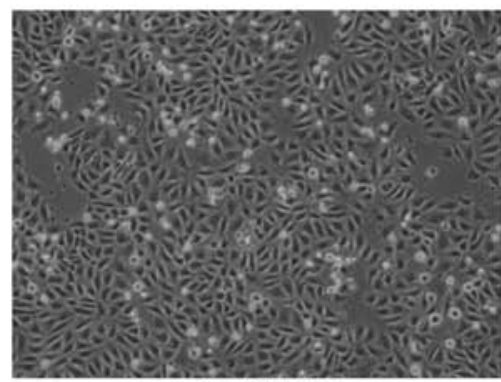

1.0 mM VPA

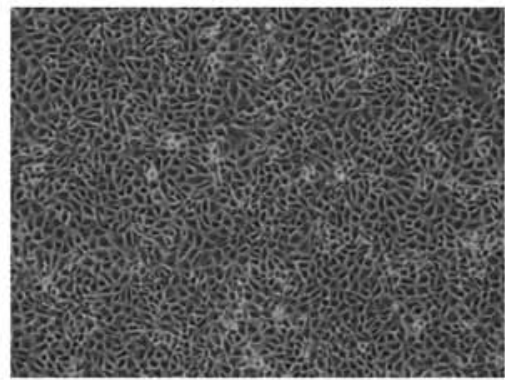

SiRNA

Figure 4. VEGI overexpression directly induces OS cell death. U-2 OS cells were transfected with $1.0 \mu \mathrm{g}$ of VEGI full-length gene expression vector or control vector or $20 \mathrm{nM}$ of siRNA for VEGI, respectively. (A) The VEGI protein expression levels were analyzed using a western blot analysis. (B) U-2 OS cells were transfected with the VEGI full-length gene expression vector (pVEGI) or siRNA for VEGI and cultured for $48 \mathrm{~h}$. The amount of soluble VEGI and DcR3 in the medium per $10^{4}$ viable cells was determined. The values were expressed as the ratio to the average amount of soluble VEGI and DcR3 per $10^{4}$ viable cells in the control cultures, set at 1 . Each bar indicates the mean $\pm \mathrm{SE}$ of values obtained from four experiments. " $\mathrm{P}<0.05$, significant difference. (C) Forty-eight hours after the transfection of pVEGI or siRNA for VEGI and 1.0 mM VPA treatment for 3 days, the U-2 OS cells were examined using an inverted microscope.

PCR analysis revealed that VEGI mRNA transcription was increased approximately 2.5-fold, although no significant difference was noted in the DR3 or DcR3 levels (Fig. 5C).

Effects of the HDAC inhibitors on the production of soluble VEGI and secretion of DcR3 in the HMVE cells. Although the HDAC inhibitors increased the soluble VEGI levels in the OS cells, we further examined the HMVE cells on day 7. Notably, increased soluble VEGI production was detected after VPA or TSA treatment (Fig. 5D). However, the DcR3 levels remained essentially unchanged by both HDAC inhibitors (Fig. 5D).

The VPA-induced soluble VEGI in the OS cells inhibits vascular tube formation. In order to confirm whether the production of soluble VEGI from the tumor cells inhibited neovascularization, we assessed the effects of OS cell culture medium treated with VPA as well as pVEGI-transfected cells. The results showed some tube formation in the HMVE cells treated with 1.0 mM VPA, although it was less than that seen 
A.

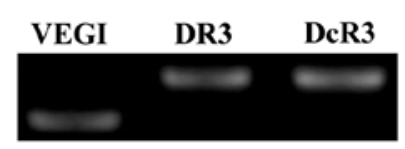

GAPDH

C.
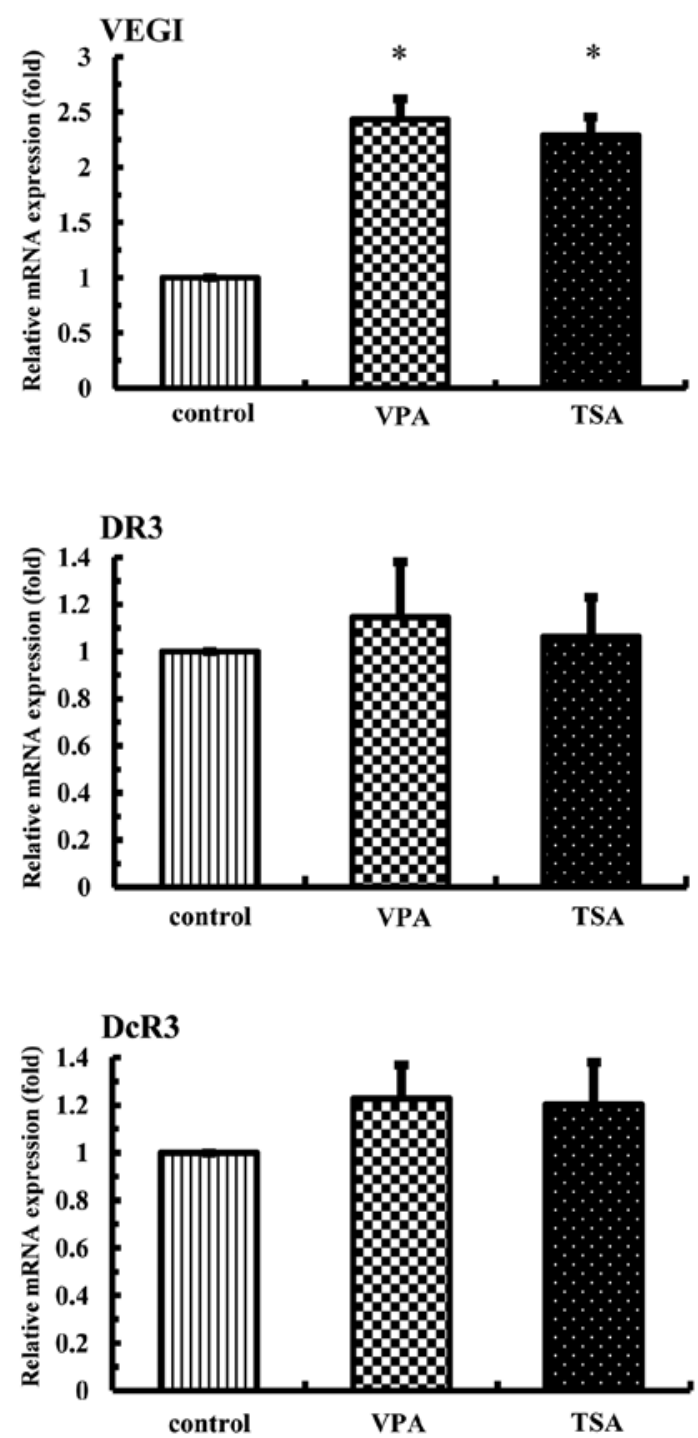

B.

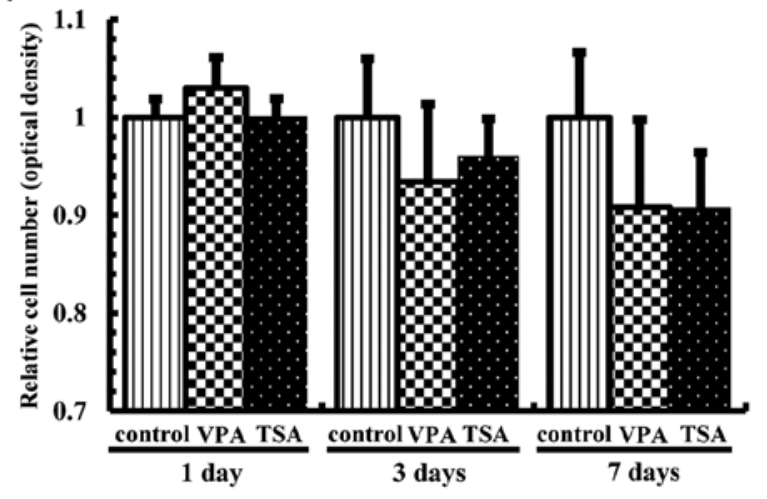

D.
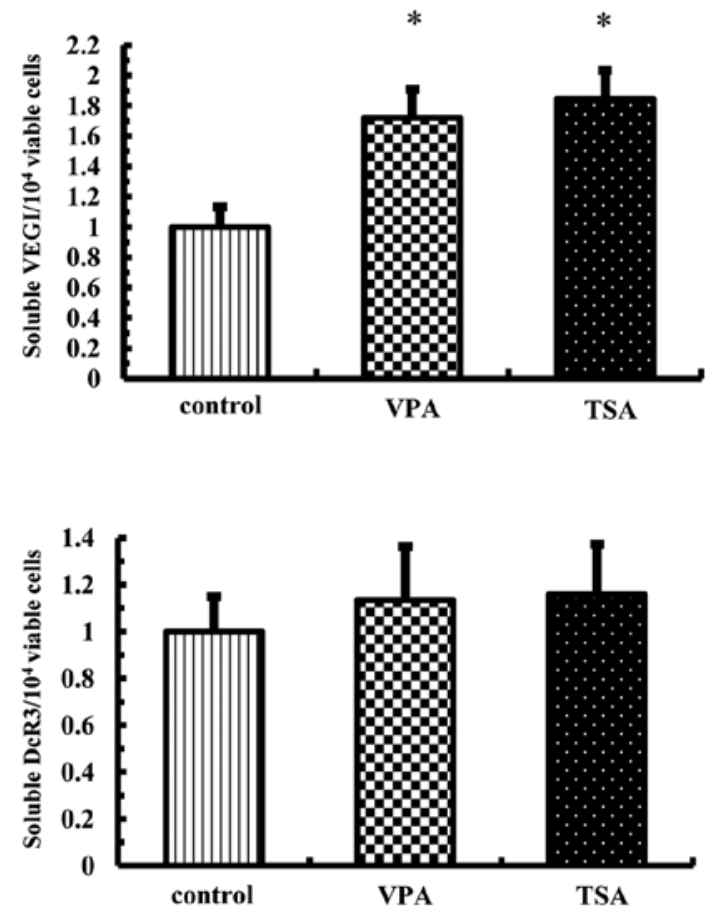

Figure 5. Effects of the HDAC inhibitors on HMVE cells. (A) Detection of the VEGI, DR3 and DcR3 mRNA transcripts in the HMVE cells using RT-PCR. (B) HMVE cells were cultured with $1.0 \mathrm{mM} \mathrm{VPA}$ and $30 \mathrm{nM}$ TSA and analyzed for growth on the indicated days. The values are presented as the ratio to the average amount of optical density in the control cultures, set at 1. Each bar indicates the mean \pm SE of 16 wells. (C) The expression levels of VEGI, DR3 and DcR3 were examined using real-time PCR. The values are expressed as the ratio to the average value in the control cultures. (D) HMVE cells were cultured in medium with or without $1.0 \mathrm{mM}$ VPA or $30 \mathrm{nM}$ TSA for 7 days, respectively. The amount of soluble VEGI and DcR3 in the medium per $10^{4}$ viable cells was determined on day 7. The values are expressed as the ratio to the average amount of soluble VEGI and DcR3 per $10^{4}$ viable cells in the control cultures, set at 1. Each bar indicates the mean $\pm \mathrm{SE}$ of values obtained from eight experiments. ${ }^{*} \mathrm{P}<0.05$, significant difference for all experiments.

in the control cells (Fig. 6B). Surprisingly, tube formation was markedly decreased by the VPA-treated OS cell culture medium (Fig. 6C), and the pVEGI-transfected medium completely suppressed tube formation (Fig. 6D).

\section{Discussion}

The present findings showed that treatment with VPA and TSA, histone deacetylase (HDAC) inhibitors, increased the expression of endogenous vascular endothelial growth inhibitor (VEGI) and its receptor, death receptor 3 (DR3), without stimulating decoy receptor 3 (DcR3) secretion, via the effects of histone acetylation. Moreover, VEGI overexpression resulted in increased OS cell death, and the VPA-treated soluble VEGI in the OS cell culture medium were associated with the inhibition of vascular tube formation in the HMVE cells. Recent studies suggest that the main role of VEGI in human cancer cells is the anticancer effect via the direct inhibition of cancer cell proliferation as well as anti-angiogenic effects on endothelial cells $(11,18)$. DR3, a member of tumor 

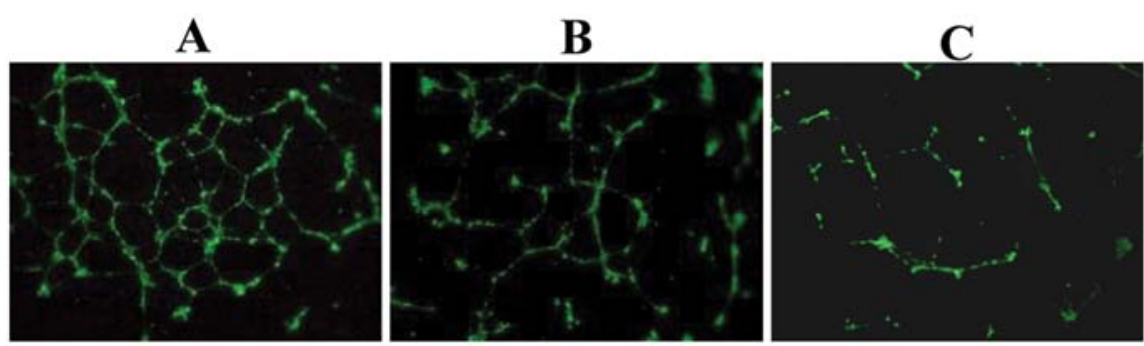

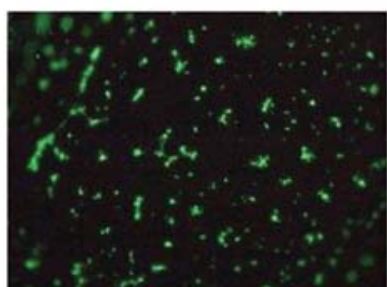

D

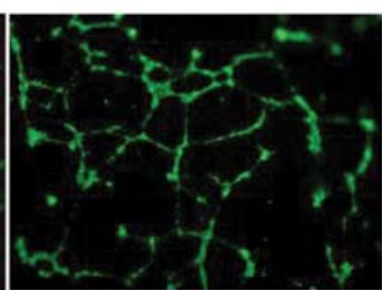

E

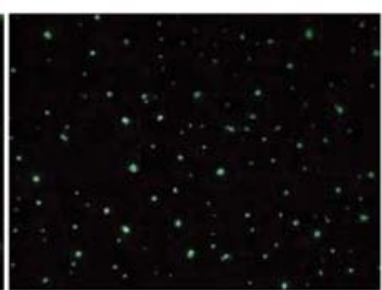

$\mathbf{F}$

Figure 6. Soluble VEGI inhibits human microvascular endothelial cell tube formation. HMVE cells were cultured or harvested under each condition: (A) control; (B) $1.0 \mathrm{mM} \mathrm{VPA}$; (C) VPA-treated OS cell culture medium; (D) pVEGI-overexpression medium; (E) siRNA-transfected medium; (F) 33 nM JNJ-10198409 (negative control), respectively. VPA-treated OS culture medium was obtained from U-2 OS cells treated with 1.0 mM VPA for 7 days and pVEGI-overexpression medium was obtained from pVEGI-transfected U-2 OS cells cultured for $48 \mathrm{~h}$. The siRNA-transfected medium was obtained from siRNA for VEGI-transfected U-2 OS cells cultured for $48 \mathrm{~h}$. A total of $33 \mathrm{nM} \mathrm{JNJ}-10198409$ were used as negative controls, respectively. The extent of endothelial cell tube formation was examined using a fluorescence microscope.

necrosis factor receptor family (TNFRSF25), has been shown to be capable of stimulating caspase-dependent cell apoptosis, and the activation of caspases contributes to VEGI-induced cell death $(8,9,11,19)$. Therefore, VEGI may serve as a potential target for the development of angiogenesis-based cancer therapy.

Although the VEGI and DR3 mRNA expression was detected in the OS cells, the corresponding proteins were expressed at low levels. This reduction in the tumor VEGI and DR3 expression suggests a shift in balance to pro-angiogenic conditions. Such loss of balance may be conducive to tumor growth and survival $(10,14)$. The VEGI expression is regulated by the central transcription factor NF- $\mathrm{BB}$ (20), and HDAC inhibition increases the NF- $\kappa \mathrm{B}$ transcriptional activity, which regulates the expression levels of a variety of genes (21). In order to recover an abnormal angiogenic condition in the tumor cells, we applied VPA and TSA as HDAC inhibitors and found that both VPA and TSA increased VEGI and DR3 mRNA transcription and also their protein expression levels. This observation indicates that HDAC inhibitors promote the recovery of angiogenic imbalance in tumor cells. The enhanced expression of DcR3 has been indicated to protect against induced apoptosis in various tumors and is positively correlated with tumor progression (22-25). Our data suggest that treatment with VPA and TSA is not accompanied by increased DcR3 secretion in OS cells. These results prompted us to explore whether VPA-induced soluble VEGI inhibits vascular tube formation in HMVE cells. Consequently, the growth of HMVE cells was not changed by VPA and TSA treatment. Although both VPA and TSA were confirmed to enhance VEGI mRNA transcription accompanied by soluble VEGI production, neither DR3 or DcR3 mRNA transcription nor soluble DcR3 production was increased. Furthermore, the effect of VPA treatment on vascular tube formation was slight compared with that observed in the control cells. Surprisingly, in vitro vascular tube formation was inhibited by the VPA-treated or VEGI-overexpressing OS cell culture conditioned media. The results showed that the soluble VEGI concentration increased $\sim 2.0$-fold by the VPA-treated OS cell medium versus that seen in the control cells and the overexpression of VEGI in the culture medium was significant. Hence, the VPA-induced soluble VEGI in OS cells induced HMVE cell death by binding of VEGI to DR3 in a paracrine manner. HDAC inhibitors have been reported to inhibit tumor angiogenesis (7). This process may be in part mediated by HIF-1 $\alpha$ downregulation in both tumor and vascular endothelial cells and the consequent downregulation of VEGF and other HIF-1 $\alpha$ regulated angiogenesis-related genes (26-29). We evaluated the effects of VPA and TSA on the VEGFA gene expression using real-time PCR. Although treatment with VPA and TSA reduced the VEGFA gene expression in both OS and HMVE cells (data not shown), the in vitro vascular tube formation assay showed that the effect of VPA on the HMVE cells was slight or limited with respect to vascular tube formation. However, the VPA-treated OS cell culture medium markedly reduced such formation, suggesting that the anti-angiogenic effect of VPA involves VEGF as well as modulation via the soluble VEGI/DR3 pathway. In addition, evidence indicates that the antitumor activity of VEGI is more likely the result of interference with the development of the tumor-associated vasculature than the direct effects on tumor cells (18-20). Our VEGI-overexpression model was found to exhibit significant OS cell death. Xiao et al, reported that the oncolytic adenovirus ZD55-VEGI-251 inhibits angiogenesis via the paracrine actions of VEGI-251 and directly induces the apoptosis of cancer cells via the autocrine actions of VEGI-251 (30). Two 
fragments of soluble VEGI are produced by ADAM17/10mediated alternative cleavage. Soluble TL1AL72-L251 acts mainly in a paracrine manner to influence cells in the immune system, whereas the TL1AV84-L251 fragment acts via the autocrine pathway to induce the inhibition of the proliferation and apoptosis of endothelial cells (31). Our previous report indicated that VPA-treated OS cells do not exhibit increased ADAM17 gene transcription (32). Hence, the increase in the expression of soluble VEGI induced by HDAC inhibitors may involve a different mechanism of action than that mediating the shedding of membrane-bound VEGI.

In conclusion, VPA has anti-angiogenesis and simultaneous antitumor activity as a result of enhancing soluble VEGI/DR3-mediated apoptosis via both autocrine and paracrine pathways. Targeted inhibition of the HDAC activity is a rational treatment that is currently in clinical development. Collectively, modulating the VEGI expression with HDAC inhibitors may eventually be exploited as a therapeutic strategy in osteosarcoma patients.

\section{Acknowledgements}

This study was supported in part by a Grant-in-Aid for Young Scientists (B) (23792159) and Grant-in-Aid for Scientific Research (C) (26462279) from the Ministry of Education, Culture, Sports, Science, and Technology of Japan, Grantin-Aid for Researchers, Hyogo College of Medicine, 2012.

\section{References}

1. Messerschmitt PJ, Garcia RM, Abdul-Karim FW, Greenfield EM and Getty PJ: Osteosarcoma. J Am Acad Orthop Surg 17: 515-527, 2009.

2. Shen $\mathrm{H}$ and Laird PW: Interplay between the cancer genome and epigenome. Cell 153: 38-55, 2013.

3. Kristensen LS, Nielsen HM and Hansen LL: Epigenetics and cancer treatment. Eur J Pharmacol 625: 131-142, 2009.

4. Ferguson LR, Tatham AL, Lin Z and Denny WA: Epigenetic regulation of gene expression as an anticancer drug target. Curr Cancer Drug Targets 11: 199-212, 2011.

5. Rogawski MA and Löscher W: The neurobiology of antiepileptic drugs. Nat Rev Neurosci 5: 553-564, 2004.

6. Bialer M: Why are antiepileptic drugs used for nonepileptic conditions? Epilepsia 53 (Suppl 7): S26-S33, 2012.

7. Dickinson M, Johnstone RW and Prince HM: Histone deacetylase inhibitors: Potential targets responsible for their anticancer effect. Invest New Drugs 28 (Suppl 1): S3-S20, 2010.

8. Tan KB, Harrop J, Reddy M, Young P, Terrett J, Emery J, Moore G and Truneh A: Characterization of a novel TNF-like ligand and recently described TNF ligand and TNF receptor superfamily genes and their constitutive and inducible expression in hematopoietic and non-hematopoietic cells. Gene 204: 35-46, 1997.

9. Zhai Y, Ni J, Jiang GW, et al: VEGI, a novel cytokine of the tumor necrosis factor family, is an angiogenesis inhibitor that suppresses the growth of colon carcinomas in vivo. FASEB J 13 : 181-189, 1999.

10. Parr C, Gan $\mathrm{CH}$, Watkins G and Jiang WG: Reduced vascular endothelial growth inhibitor (VEGI) expression is associated with poor prognosis in breast cancer patients. Angiogenesis 9: 73-81, 2006.

11. Chew LJ, Pan H, Yu J, Tian S, Huang WQ, Zhang JY, Pang S and Li LY: A novel secreted splice variant of vascular endothelial cell growth inhibitor. FASEB J 16: 742-744, 2002.

12. Migone TS, Zhang J, Luo X, Zhuang L, Chen C, Hu B, Hong JS, Perry JW, Chen S-F and Zhou JXH: TL1A is a TNF-like ligand for DR3 and TR6/DcR3 and functions as a T cell costimulator. Immunity 16: 479-492, 2002.
13. Grimaldo S, Tian F and Li LY: Sensitization of endothelial cells to VEGI-induced apoptosis by inhibiting the NF-kappaB pathway. Apoptosis 14: 788-795, 2009.

14. Ge Z, Sanders AJ, Ye L and Jiang WG: Aberrant expression and function of death receptor-3 and death decoy receptor-3 in human cancer. Exp Ther Med 2: 167-172, 2011.

15. Liang PH, Tian F, Lu Y, Duan B, Stolz DB and Li LY: Vascular endothelial growth inhibitor (VEGI; TNFSF15) inhibits bone marrow-derived endothelial progenitor cell incorporation into Lewis lung carcinoma tumors. Angiogenesis 14: 61-68, 2011.

16. Chinnaiyan AM, O'Rourke K, Yu GL, Lyons RH, Garg M, Duan DR, Xing L, Gentz R, Ni J and Dixit VM: Signal transduction by DR3, a death domain-containing receptor related to TNFR-1 and CD95. Science 274: 990-992, 1996.

17. Yang CR, Hsieh SL, Teng CM, Ho FM, Su WL and Lin WW: Soluble decoy receptor 3 induces angiogenesis by neutralization of TL1A, a cytokine belonging to tumor necrosis factor superfamily and exhibiting angiostatic action. Cancer Res 64: 1122-1129, 2004

18. Hou W, Medynski D, Wu S, Lin X and Li LY: VEGI-192, a new isoform of TNFSF15, specifically eliminates tumor vascular endothelial cells and suppresses tumor growth. Clin Cancer Res 11: 5595-5602, 2005.

19. Zhang $Z$ and Li LY: TNFSF15 modulates neovascularization and inflammation. Cancer Microenviron 5: 237-247, 2012.

20. Xiao Q,Hsu CY,Chen H, Ma X, Xu J and Lee JM: Characterization of cis-regulatory elements of the vascular endothelial growth inhibitor gene promoter. Biochem J 388: 913-920, 2005.

21. Marks PA and Xu WS: Histone deacetylase inhibitors: Potential in cancer therapy. J Cell Biochem 107: 600-608, 2009.

22. Bai C, Connolly B, Metzker ML, et al: Overexpression of M68/DcR3 in human gastrointestinal tract tumors independent of gene amplification and its location in a four-gene cluster. Proc Natl Acad Sci USA 97: 1230-1235, 2000.

23. Takahama Y, Yamada Y, Emoto K, Fujimoto H, Takayama T, Ueno M, Uchida H, Hirao S, Mizuno T and Nakajima Y: The prognostic significance of overexpression of the decoy receptor for Fas ligand (DcR3) in patients with gastric carcinomas. Gastric Cancer 5: 61-68, 2002

24. Ge Z, Sanders AJ, Ye L, Wang Y and Jiang WG: Expression of death decoy receptor-3 (DcR3) in human breast cancer and its functional effects on breast cancer cells in vitro. J Exp Ther Oncol 9: 109-118, 2011.

25. Zong L, Chen P and Wang DX: Death decoy receptor overexpression and increased malignancy risk in colorectal cancer. World $\mathrm{J}$ Gastroenterol 20: 4440-4445, 2014.

26. Qian DZ, Kachhap SK, Collis SJ, Verheul HM, Carducci MA, Atadja P and Pili R: Class II histone deacetylases are associated with VHL-independent regulation of hypoxia-inducible factor 1 alpha. Cancer Res 66: 8814-8821, 2006.

27. Kong X, Lin Z, Liang D, Fath D, Sang N and Caro J: Histone deacetylase inhibitors induce VHL and ubiquitin-independent proteasomal degradation of hypoxia-inducible factor 1alpha. Mol Cell Biol 26: 2019-2028, 2006.

28. Kang FW, Que L, Wu M, Wang ZL and Sun J: Effects of trichostatin A on HIF-1 $\alpha$ and VEGF expression in human tongue squamous cell carcinoma cells in vitro. Oncol Rep 28: 193-199, 2012.

29. Zhao Y, Yu D, Wu H, Liu H, Zhou H, Gu R, Zhang R, Zhang S and Wu G: Anticancer activity of SAHA, a potent histone deacetylase inhibitor, in NCI-H460 human large-cell lung carcinoma cells in vitro and in vivo. Int J Oncol 44: 451-458, 2014.

30. Xiao T, Fan JK, Huang HL, Gu JF, Li LY and Liu XY: VEGI-armed oncolytic adenovirus inhibits tumor neovascularization and directly induces mitochondria-mediated cancer cell apoptosis. Cell Res 20: 367-378, 2010.

31. Mück C, Herndler-Brandstetter D, Micutkova L, GrubeckLoebenstein B and Jansen-Dürr P: Two functionally distinct isoforms of TL1A (TNFSF15) generated by differential ectodomain shedding. J Gerontol A Biol Sci Med Sci 65: 1165-1180, 2010.

32. Yamanegi K, Yamane J, Kobayashi K, et al: Downregulation of matrix metalloproteinase- 9 mRNA by valproic acid plays a role in inhibiting the shedding of MHC class I-related molecules A and B on the surface of human osteosarcoma cells. Oncol Rep 28: 1585-1590, 2012. 